\title{
The "Ground-Glass" Mimicker in The Pandemic: A Novel Radiomics-Based Machine Learning Model Differentiates COVID-19 Pneumonia from Acute Non-COVID-19 Lung Disease
}

\section{Andrea Delli Pizzi}

Department of Neuroscience, Imaging and Clinical Sciences, "G. d'Annunzio" University, Chieti Antonio Chiarelli

Department of Neuroscience, Imaging and Clinical Sciences, "G. d'Annunzio" University, Chieti Piero Chiacchiaretta ( $\nabla$ p.chiacchiaretta@unich.it)

Department of Neuroscience, Imaging and Clinical Sciences, “G. d'Annunzio" University, Chieti Cristina Valdesi

Department of Neuroscience, Imaging and Clinical Sciences, “G. d'Annunzio" University, Chieti

\section{Pierpaolo Croce}

Department of Neuroscience, Imaging and Clinical Sciences, "G. d'Annunzio" University, Chieti

\section{Domenico Mastrodicasa}

Stanford University School of Medicine, Department of Radiology, Stanford, CA

\section{Michela Villani}

Department of Neuroscience, Imaging and Clinical Sciences, "G. d'Annunzio" University, Chieti

\section{Stefano Trebeschi}

Department of Radiology, Netherlands Cancer Institute, Amsterdam

\section{Francesco Serafini}

Department of Neuroscience, Imaging and Clinical Sciences, "G. d'Annunzio" University, Chieti

\section{Consuelo Rosa}

Department of Radiation Oncology, "Santissima Annunziata” Hospital, “G. D’Annunzio" University of Chieti, Via Dei Vestini, 66100 Chieti

\section{Giulio Cocco}

Unit of Ultrasound in Internal Medicine, Department of Medicine and Science of Aging, "G. D'Annunzio" University, Chieti

\section{Riccardo Luberti}

Department of Neuroscience, Imaging and Clinical Sciences, "G. d'Annunzio" University, Chieti

\section{Sabrina Conte}

Department of Neuroscience, Imaging and Clinical Sciences, "G. d'Annunzio" University, Chieti

\section{Lucia Mazzamurro}

Department of Neuroscience, Imaging and Clinical Sciences, "G. d'Annunzio" University, Chieti 


\section{Manuela Mereu}

Department of Neuroscience, Imaging and Clinical Sciences, "G. d'Annunzio" University, Chieti

\section{Rosa Patea}

Department of Neuroscience, Imaging and Clinical Sciences, “G. d'Annunzio" University, Chieti

\section{Valentina Panara}

Department of Neuroscience, Imaging and Clinical Sciences, "G. d'Annunzio" University, Chieti

\section{Stefano Marinari}

Department of Pneumology, "Santissima Annunziata” Hospital, “G. D’Annunzio" University of Chieti, Via Dei Vestini, 66100 Chieti

\section{Jacopo Vecchiett}

Clinic of Infectious Diseases, Department of Medicine and Science of Aging, University 'G. d'Annunzio' Chieti-Pescara, Chieti

\section{Massimo Caulo}

Department of Neuroscience, Imaging and Clinical Sciences, “G. d’Annunzio” University, Chieti

\section{Research Article}

Keywords: HRCT, Ground Glass Opacities, Radiomics, Machine Learning, COVID-19, Differential Diagnosis

Posted Date: December 10th, 2020

DOI: https://doi.org/10.21203/rs.3.rs-115659/v1

License: (c) (1) This work is licensed under a Creative Commons Attribution 4.0 International License. Read Full License 


\section{Abstract}

Ground-Glass Opacities (GGOs) are a non-specific CT finding observed in the early phase of COVID-19 pneumonia. However, GGOs are also seen in other acute interstitial and alveolar lung diseases, thus making the differential diagnosis a diagnostic challenge. In this poof-of-concept study, we aimed to differentiate COVID-19 pneumonia presenting with GGOs from acute non-COVID-19 lung disease using a novel radiomic-based model in patients who underwent a high-resolution CT (HRCT) scan at hospital admission during the first pandemic peak in Italy. HRCT scans of 28 RT-PCR diagnosed COVID-19 pneumonia (COVID) and 30 acute non-COVID-lung disease (nCOVID) were retrospectively included. All patients showed GGOs as the predominant CT pattern. Two readers, blinded to the final diagnosis, independently segmented GGOs on CT scans by using a semi-automated approach, and radiomic features were extracted from segmented images. Partial least square (PLS) regression was used as the multivariate machine-learning algorithm. A leave-one-out nested cross-validation was implemented to optimize the hyperparameter of PLS and to assess the model generalization. The diagnostic performance of the radiomic model to differentiate between COVID and nCOVID lung disease was assessed through receiver operating characteristic (ROC) analysis. The radiomics-based machine learning model differentiated COVID and nCOVID with an AUC $=0.868\left(p=4 \cdot 2 \cdot 10^{-7}\right)$. After a careful prospective evaluation in larger multicentric studies, it may help radiologists to rule out COVID-19 pneumonia thus improving the COVID-19 triaging in epidemic areas.

\section{Introduction}

COVID-19 is a viral infectious disease caused by SARS-CoV-2, which has gradually spread worldwide since December $2019(1,2)$. The clinical presentation is extremely varied and ranges from asymptomatic or paucisymptomatic infection to severe pneumonia with respiratory failure $(3,4)$. COVID-19 pneumonia is caused by partial filling of airspaces, interstitial thickening, partial collapse of alveoli, increased capillary blood volume, or a combination of these elements (5). This histologic scenario reflects different CT patterns of presentation, including GGOs with or without superimposed septal thickening ("crazy paving" pattern), and parenchymal consolidations (6-10). However, GGOs are a non-specific CT finding. In fact, they can be found in the early, exudative phase of COVID-19 pneumonia as well as in interstitial and alveolar diseases, such as pulmonary edema, alveolar hemorrhage, infectious pneumonia, hypersensitivity pneumonia, and others acute lung diseases (11-13). Although the context of the current pandemic and the previous CT findings can be indicative of COVID-19 pneumonia, the differential diagnosis remains a challenge $(14,15)$. Integration with clinical data, laboratory tests, and lung biopsy may be necessary when the initial diagnosis is inconclusive (16). Radiomics is a recently developed method that allows for extracting large amounts of quantitative data features from medical images (17). A typical radiomic analysis includes evaluating the size, shape, and textural features containing spatial information on pixel or voxel intensity distribution and patterns. Radiomic features can be further integrated into machine learning models with the aim to improve diagnosis and patient management. This approach was recently proposed in oncology with promising results (18-21). For example, a 
framework combining radiomics and deep learning recently predicted high-grade lung adenocarcinoma (19). Moreover, a radiomic model was demonstrated to predict the tumor invasiveness of pulmonary adenocarcinomas appearing as ground-glass nodules $(18,21)$. Radiomics-based models were recently proposed to improve the diagnosis of COVID-19 on chest CT images (22-24). To the best of our knowledge, no studies investigated the possibility of discriminating the GGOs due to COVID-19 pneumonia from those due to non-COVID-19 acute lung disease.

In this study, we aimed to differentiate on CT scans COVID-19 pneumonia presenting with GGOs, acquired at hospital admission during the first pandemic peak, from acute non-COVID-19 lung disease using a novel radiomic-based model.

\section{Materials And Methods}

Study Population. The study received formal approval from the Ethical Committee of the University G. D'Annunzio of Chieti-Pescara, Italy; informed consent was waived by the same ethics committee that approved the study (Comitato Etico per la Ricerca Biomedica delle Province di Chieti e Pescara e dell'Università degli Studi "G. d'Annunzio" di Chieti e Pescara, Italy). The study was conducted according to ethical principles laid down by the latest version of the Declaration of Helsinki. We retrospectively included a total of 120 consecutive patients diagnosed with SARS-CoV-2 infection based on RT-PCR who underwent a clinically indicated high-resolution chest CT (HRCT) between March 2020 and April 2020. Patients were included if they met all the following criteria: (a) GGO as predominant feature on chest CT scans, (b) baseline HRCT performed at hospital admission. Another set of 310 patients (nCOVID) with clinically indicated HRCT for acute respiratory disease performed between August 2019 and April 2020 was retrospectively enrolled in the study (nCOVID). For this second set, patients were included if they met all the following criteria: (a) GGO as predominant feature on chest CT scans, (b) availability of final diagnosis (clinical, laboratory, or pathology). In the first set (COVID), we excluded 92 patients: 14 had severe respiratory artefacts, 92 had a non-predominant GGO pattern. In the second set (nCOVID) we excluded 280 patients: 32 had severe respiratory artefacts, 210 had a non-predominant GGO alteration and 38 were treated in another center and the final diagnosis was not available. The final study population was composed of 28 COVID and 30 nCOVID for a total of 58 patients (Fig. 1).

CT Protocol. Non-enhanced chest CT scans were performed in a supine position, during inspiratory breath-hold, from the apex to the lung bases, with a 128-slice multi-detector CT device (Somatom Definition AS, Siemens Healthineers, Germany). The field of view (FOV) ranged between $35-40 \mathrm{~cm}$ according to the body size. The electronic window values were amplitude (W) $1200-1600 \mathrm{UH}$ and window or center level $(\mathrm{L})$ between -600 and $-750 \mathrm{UH}$. The main scan parameters were: tube voltage $=$ $120 \mathrm{kVp}$, automatic tube current modulation (30-70 mAs), pitch $=0.99-1.22 \mathrm{~mm}$, matrix $=512 \times 512$. The images were reconstructed with a slice thickness of $0.625-1.250 \mathrm{~mm}$ with the same increment with a high spatial frequency reconstruction algorithm (B50, I50). 
Radiomics Analysis. A whole-volume semi-automated GGOs delineation was independently performed by two senior radiology residents (C.V. and M.V.) using an open-source medical image computing platform, 3DSlicer Version 4.8 (www.3dslicer.org) (Fig. 2a). The GGO threshold was manually set between - 1350 and $-700 \mathrm{HU}$ using the "threshold-effect" tool $(9,25,26)$. If necessary, the segmentation was further manually corrected by each reader in order to exclude automated segmented pixels beyond the GGOs. Moreover, the lungs were automatically extracted via Convolutional Neural Network (CNN) algorithms to create binary mask (27). Then, a logical "and", between these masks and the segmentations obtained by the radiology residents, was performed (using "3dcalc") to exclude automated segmented pixels beyond the lungs, thus obtaining the final ROIs (28). All the ROls were then finally checked by a radiologist with more than 10 years of experience in chest imaging (M.M.) to verify the correct position and correspondence with the underlying CT images. The reproducibility assessment of the features extracted by the two independent segmentation sets of the 58 CT scans (28 COVID, $30 \mathrm{nCOVID}$ ) was performed. The extraction of the radiomic features was conducted using PyRadiomics, a flexible open-source platform capable of extracting a large panel of engineered features from medical images; this radiomic quantification platform enables the standardization of both feature definitions and image processing (29). To avoid data heterogeneity bias, HRCT images were subjected to imaging resampling $(2 \times 2 \times$ $2 \mathrm{~mm}$ ). For each ROI, ten built-in filters (Original, wavelet, Laplacian of Gaussian (LoG), square, square root, logarithm, exponential, Gradient, LBP2D, LBP3D) were applied and seven feature classes (first order statistics, shape descriptors, glcm, glrlm, ngtdm, gldm, glszm) were calculated, for a total of 1409 radiomic features.

Machine Learning Approach: Partial Least Square (pls) Regression

A Machine learning (i.e. multivariate) approach was implemented to exploit radiomic features multidimensionality (Fig. 2b). When trying to predict an output based on these features, the information redundancy (i.e., radiomic features high correlation), coupled with a low number of independent samples (i.e., subjects), makes the prediction unstable to noise and prone to poor generalization $(30,31)$. To address this problem, two main approaches were implemented. The first approach was to reduce the number of features by selecting only those that were highly repeatable $(r>0.95)$ between the two masks (delineated by the two radiologists). The second approach was to implement a machine learning framework based on a linear regression analysis that employed a space dimension reduction procedure, namely the partial least square (PLS) regression $(30,32,33)$. The PLS was used to differentiate COVID from nCOVID patients. PLS allows the construction of regression equations reducing the predictors to a smaller set of uncorrelated components, i.e. a linear combination of the original predictors, and performs regression on these components $(33,34)$. The goal of PLS is to identify components that capture most of the information in the independent variables (e.g., linear combinations of all radiomic features) that is useful for predicting the dependent variable (e.g., COVID vs. nCOVID). PLS is the supervised learning version of the Principal Component Analysis (PCA) $(35,36)$. The learning process (fitting) of the PLS algorithm delivers regression loadings that can be used to retrieve the weights ( $\beta$-weights) linking the original independent variables with the dependent variable, depicting the importance and sign of the original variables in the prediction. The PLS has one hyperparameter to be optimized, namely the number 
of uncorrelated components to be used in the regression. To perform hyperparameter optimization and evaluate the generalizable performance of the procedure an approach that allows to minimize the loss of samples during training of the model is the nested cross-validation (nCV) (37). In nCV, data are divided in folds and the model is trained on all data except one-fold in an iterative, nested manner. The hyperparameter optimization and performance assessment are performed on the remaining fold and averaged across iterations. If the number of folds equals the number of samples (one-fold per sample) the procedure is defined leave-one-out nCV $(38,39)$. This approach is highly suited for medical applications where each sample represents one subject. In this work, a leave-one-out nCV was implemented to optimize the PLS number of components and to assess the PLS generalization performance. The $\beta$-weights of the PLS analysis were obtained by running the algorithm on the complete dataset with the optimal number of components delivered by the $\mathrm{nCV}$ analysis. The machine learning analyses were implemented in Matlab.

\section{Statistical Analysis}

The COVID vs nCOVID classification performance was assessed through Receiver Operating Characteristic (ROC) analysis comparing the inferred (out-of-training-sample) with the true group. COVID patients were attributed to the "positive" group, whereas nCOVID patients were attributed to the "negative" group. The ROC analysis was also performed on random shuffled group labels to simulate the null hypothesis and evaluate its confidence interval (repeated $10^{6}$ times). The ROC analysis delivered an Area Under the Curve (AUC), which could be transformed into a z-score for assessing its statistical significance by using the random shuffled group labels. The statistical analysis was performed in Matlab.

\section{Results}

The majority of patients included in the study were male $(n=34,59 \%)$, and the median age was 66 years (interquartile range 55-81). Out of the total patient population $(n=58), 28(48 \%)$ were assigned to the COVID group, and $30(52 \%)$ to the nCOVID group. The nCOVID group $(n=30)$ included four with Cytomegalovirus (CMV) pneumonia, two with pulmonary edema, five with Acute Respiratory Distress Syndrome (ARDS), eight with Organizing Pneumonia, three with Pneumocystis Jirovecii pneumonia, two with Influenza A pneumonia, two with Legionella pneumonia, three with alveolar hemorrhage and one with hypersensitivity pneumonia (Table 1). A total of 1409 radiomic features were extracted. 153 of these features showed an inter-reader correlation of $r>0.95$ and were used for further analysis. When employing radiomic features with an $r>0.95$, i.e., 153 radiomic features, an AUC $=0.868$ was obtained ( $z$ $=5.1, p=4 \cdot 2 \cdot 10^{-7}$, Fig. 3). The estimated optimal number of PLS components, evaluated within the nCV framework, was 7. The weights of the PLS ( $\beta$-weights) are shown in Fig. 4. Figure 4a reports the distributions of the $\beta$-weights for radiomic features used in the analysis, whereas Fig. $4 \mathrm{~b}$ depicts the $\beta$ weights associated to the top $5 \%$ of the selected features with the largest $\beta$-weights in magnitude, i.e. those most impacting the prediction. Of the top $5 \%$ features, 5

(wavelet_LLH_glrlm_GrayLevelNonUniformity, wavelet_LHH_glcm_DifferenceVariance, 
wavelet_LHH_glrlm_GrayLevelVariance, wavelet_HLH_glcm_DifferenceVariance, wavelet_HHL_glrlm_RunEntropy), were associated to glrlm and glcm texture matrices (second second order features), and 2 (wavelet_LLH_firstorder_Skewness, Ibp_2D_firstorder_10Percentile) were related to the image intensity distribution (first order features). All, except one, second order features had a negative weight, meaning that COVID-19 patients (labelled as 1 in the classification algorithm) tended to have a more homogeneous texture. Of the two first order features, one had positive weight (the skweness, larger value in COVID-19 patients) and one had a negative weight (the 10th percentile, smaller value in COVID19 patients) indicating that COVID group, although having a distribution of image intensities with average equal values that nCOVID group, had a larger occurrence of low intensity pixels.

Table 1

Descriptive baseline characteristics of our study population $(n=58)$.

\begin{tabular}{|c|c|c|}
\hline \multicolumn{2}{|l|}{ Variable } & \multirow{2}{*}{$\begin{array}{l}\text { Value } \\
34(59 \%)\end{array}$} \\
\hline Gender & Male & \\
\hline & Female & $24(41 \%)$ \\
\hline \multicolumn{2}{|c|}{ Median Age (IQR*) } & $66(55-81)$ \\
\hline \multicolumn{2}{|c|}{$\mathrm{HRCT}^{\star}$ Exam } & 58 \\
\hline nCOVID & Citomegalovirus (CMV) Pneumonia & $4(6.9 \%)$ \\
\hline \multirow[t]{8}{*}{ Group } & Pulmonary Edema & $2(3.4 \%)$ \\
\hline & Acute Distress Respiratory Syndrome (ARDS) & $5(8.6 \%)$ \\
\hline & Organizing Pneumonia & $8(13.8 \%)$ \\
\hline & Alveolar Hemorrage & $3(5.2 \%)$ \\
\hline & Hypersensitivity Pneumonia & $1(1.7 \%)$ \\
\hline & Influenza A Pneumonia & $2(3.4 \%)$ \\
\hline & Legionella Pneumonia & $2(3.4 \%)$ \\
\hline & Pneumocysisi Jirovecii Pneumonia & $3(5.2 \%)$ \\
\hline COVID & COVID-19 Pneumonia & $28(48.4 \%)$ \\
\hline \multicolumn{3}{|l|}{ Group } \\
\hline$*=I Q R \ln t$ & -Quartile Range; HRCT High-Resolution CT & \\
\hline
\end{tabular}

\section{Discussion}

Our results showed that a radiomics-based machine learning model has the potential to differentiate the GGOs due to COVID-19 pneumonia from the GGOs associated with non-COVID-19 lung diseases. Two are 
the major clinical impications of our study. First, due to its high sensitivity, CT has been proposed as the primary diagnostic tool in epidemic areas $(13,40)$. Moreover, the WHO recommended chest imaging for the diagnostic workup of symptomatic patients with suspected COVID-19 if RT-PCR testing was unavailable or delayed or when initial RT-PCR testing was negative but patients had high clinical suspicion of COVID-19 (41). In this context, the early identification of the GGO etiology could help to promptly adopt the appropriate management. For instance, patients admitted at the hospital for suspected COVID-19 pneumonia are temporarily placed in dedicated COVID-19 rule-out units, and they may experience a delay in care or intervention (14). In this scenario, chest CT is used as a surrogate for the early identification of COVID-19 pneumonia may help to triage activity, both by identifying an alternative diagnosis and by improving the patient selection for intensive/non-intensive care in case of clinical worsening. Secondly, the treatment could differ. For example, patients with organizing pneumonia are usually treated with corticosteroid therapy with the occasional addition of antibiotics (42). On the other hand, corticosteroids are recommended only in patients with severe and critical COVID-19 infection (43).

Other radiomics-based studies have already tested the differential diagnosis of GGOs; however, they were conducted in the oncology field and took place in the pre-covid era $(18,44)$. For example, Li et al. found that the voxel count and correlation features were significant differentiators of preinvasive lesions from invasive pulmonary adenocarcinoma and mini-invasive adenocarcinoma (44). Similarly, Xue et al. demonstrated that pure or mixed lesions and fractal dimension were predictors of invasive adenocarcinomas appearing as GGO (18). Chae et al. proposed a computerized texture analysis of GGOs showing that the mass and the kurtosis were significant differentiators of pre-invasive lesions from invasive pulmonary adenocarcinoma (45).

Interestingly, most of the features useful to the differential diagnosis regarded the texture analysis and revealed a higher homogeneity in COVID-19 than in non-COVID-19 patients. To the best of our knowledge, our study is the first attempt to explore the differentiation of COVID-19 pneumonia with predominant GGO pattern with radiomics. Further studies are necessary to explore if and how the texture heterogeneity is related to the COVID-19 pathophysiology. In this regard, recent studies focused on lung oncology demonstrated that an increased degree of heterogeneity is associated with malignant lung cancer in GGO $(18,21)$. We speculated that the higher homogeneity in COVID-19 pneumonia may reflect the degree of inflammatory infiltrate in the early stage of diffuse alveolar damage. In fact, GGOs are typically observed in the exudative phase of COVID-19 pneumonia, which is characterized by interstitial and alveolar oedema, hemorrhage, and hyaline membrane formation. With the progression of the disease (progressive stage), GGOs increase in density and heterogeneity, thus evolving in a more consolidative pattern or with a "crazy paving" pattern (13).

Although our results are promising, there are some limitations. Firstly, our study included a relatively low number of patients. However, our investigation was intended as a proof-of-concept study, and our aim was to explore the feasibility of a radiomics-based model to differentiate COVID-19 pneumonia from other acute lung diseases sharing the GGO as predominant CT pattern. Moreover, since our study was 
focused on GGO, our inclusion criteria were necessarily strict thus considering only COVID-19 patients with GGO as predominant pattern. In addition, GGOs are typically found in the acute phase of the disease, which may not correspond with the timing of CT. In fact, CT timing is widely influenced by the patient clinical condition and the treatment response. Secondly, compared to the number of patients included in the study, we analyzed a large number of predictive features. Indeed, the PLS algorithm, thanks to its ability to exploit the high collinearity of the different radiomic features, was nonetheless able to deliver a high prediction performance. However, we expect that by reducing the ratio between features and subject, the model prediction may further increase. Thirdly, this is a retrospectively designed, single-center study. Further prospective and possibly multicentric studies are warranted to define a more standardized approach.

In this proof-of-concept study, we showed that a radiomics-based machine learning model can reliably differentiate COVID-19 pneumonia-related GGOs from GGOs due to other acute lung diseases. This approach was tested on CT exams obtained at the time of hospital admission during the pandemic peak. Therefore, after a careful prospective evaluation in larger multicentric studies, it may help radiologists to rule out COVID-19 pneumonia thus improving the COVID-19 triaging in epidemic areas.

\section{Abbreviations}

AUC Area under the curve

COVID-19 Coronavirus disease 2019

HRCT High-resolution Computed Tomography

ROC Receiver operating characteristic curve

RT-PCR Real-time polymerase chain reaction

SARS-CoV-2 Severe acute respiratory syndrome coronavirus 2

\section{Declarations}

Ethical statement. This study was approved by the local ethics committee. The study used only preexisting medical data, therefore patient consent was waived.

Data Availability. The datasets generated during and/or analyzed during the current study are not publicly available due to the clinical and confidential nature of the material but can be made available from the corresponding author on reasonable request.

Author Contributions A.D.P. conceived and developed the research idea with the assistance of S.T., M.M. and M.C. The study design was performed by A.D.P, S.T. and M.C. M.V. and S.C. performed the segmentation task. R.L.P., S.M., J.V., M.V., V.P., L.M., F.L.S., C.V., R.L., C.R. and G.C. performed data 
collection. P.CR., P.CH. and A.M.C. performed the computational experiments. The paper was written by A.D.P. with technical content from A.M.C., P.CH and D.M., and extensive editorial input from all authors. All authors have read and approved the final version submitted.

\section{Competing Interests:}

DM - Disclosures: Activities related to the present article: none. Activities not related to the present article: shareholder of Segmed, Inc. Consultant for Segmed, Inc.

The other authors declared no competing interests.

\section{References}

1. Madabhavi I, Sarkar M, Kadakol N. CoviD-19: A review. Monaldi Archives for Chest Disease. 2020;90(2):248-58.

2. Rothan HA, Byrareddy SN. The epidemeology and pathogensis of coronavirus (Covid-19) outbreak. Journal of Autoimmunity. 2020;109(January):1-4.

3. Pascarella G, Strumia A, Piliego C, Bruno F, Del Buono R, Costa F, et al. COVID-19 diagnosis and management: a comprehensive review. Journal of Internal Medicine. 2020;288(2):192-206.

4. Zhao W, Zhong Z, Xie X, Yu Q, Liu J. Relation between chest CT findings and clinical conditions of coronavirus disease (covid-19) pneumonia: A multicenter study. American Journal of Roentgenology. 2020;214(5):1072-7.

5. Miller WT, Shah RM. Isolated diffuse ground-glass opacity in thoracic CT: Causes and clinical presentations. American Journal of Roentgenology. 2005;184(2):613-22.

6. Akçay Ş, Özlü T, Yilmaz A. Radiological approaches to covid-19 pneumonia. Turkish Journal of Medical Sciences. 2020;50(SI-1):604-10.

7. Hani C, Trieu NH, Saab I, Dangeard S, Bennani S, Chassagnon G, et al. COVID-19 pneumonia: A review of typical CT findings and differential diagnosis. Diagnostic and Interventional Imaging. 2020;101(5):263-8.

8. Hansell DM, Bankier AA, MacMahon H, McLoud TC, Müller NL, Remy J. Fleischner Society: Glossary of terms for thoracic imaging. 2008.

9. Ohkubo H, Kanemitsu Y, Uemura T, Takakuwa O, Takemura M, Maeno K, et al. Normal lung quantification in usual interstitial pneumonia pattern: The impact of threshold-based volumetric CT analysis for the staging of idiopathic pulmonary fibrosis. PLoS ONE. 2016;11(3):1-13.

10. Shi H, Han X, Jiang N, Cao Y, Alwalid O, Gu J, et al. Radiological findings from 81 patients with COVID-19 pneumonia in Wuhan, China: a descriptive study. The Lancet Infectious Diseases. 2020;20(4):425-34.

11. Caruso D, Zerunian M, Polici M, Pucciarelli F, Polidori T, Rucci C, et al. Chest CT Features of COVID-19 in Rome, Italy. Radiology. 2020;296(2):E79-E85.

12. Rossi SE, Erasmus JJ, Volpacchio M, Franquet T, Castiglioni T, Page McAdams H. "Crazy-Paving" Pattern at Thin-Section CT of the Lungs: Radiologic-Pathologic Overview. Radiographics. 
2003;23(6):1509-19.

13. Larici AR, Cicchetti G, Marano R, Merlino B, Elia L, Calandriello L, et al. Multimodality imaging of COVID-19 pneumonia: from diagnosis to follow-up. A comprehensive review. Eur J Radiol. 2020;131:109217.

14. Parekh M, Donuru A, Balasubramanya R, Kapur S. Review of the Chest CT Differential Diagnosis of Ground-Glass Opacities in the COVID Era. Radiology. 2020.

15. Duzgun SA, Durhan G, Demirkazik FB, Akpinar MG, Ariyurek OM. COVID-19 pneumonia: the great radiological mimicker. Insights Imaging. 2020;11(1):118.

16. Parekh M, Donuru A, Balasubramanya R, Kapur S. Review of the Chest CT Differential Diagnosis of Ground-Glass Opacities in the COVID Era. Radiology. 2020:202504.

17. Koçak B, Durmaz EŞ, Ateş E, Kılıçkesmez Ö. Radiomics with artifıcial intelligence: A practical guide for beginners. Diagnostic and Interventional Radiology. 2019;25(6):485-95.

18. Xue X, Yang Y, Huang Q, Cui F, Lian Y, Zhang S, et al. Use of a Radiomics Model to Predict Tumor Invasiveness of Pulmonary Adenocarcinomas Appearing as Pulmonary Ground-Glass Nodules. BioMed Research International. 2018.

19. Wang X, Zhang L, Yang X, Tang L, Zhao J, Chen G, et al. Deep learning combined with radiomics may optimize the prediction in differentiating high-grade lung adenocarcinomas in ground glass opacity lesions on CT scans. European Journal of Radiology. 2020;129(June):109150-.

20. Trebeschi S, Drago SG, Birkbak NJ, Kurilova I, Calin AM, Delli Pizzi A, et al. Predicting response to cancer immunotherapy using noninvasive radiomic biomarkers. Ann Oncol. 2019;30(6):998-1004.

21. Luo T, Xu K, Zhang Z, Zhang L, Wu S. Radiomic features from computed tomography to differentiate invasive pulmonary adenocarcinomas from non-invasive pulmonary adenocarcinomas appearing as part-solid ground-glass nodules. Chinese Journal of Cancer Research. 2019;31(2):329-38.

22. Zeng QQ, Zheng KI, Chen J, Jiang ZH, Tian T, Wang XB, et al. Radiomics-based model for accurately distinguishing between severe acute respiratory syndrome associated coronavirus 2 (SARS-CoV-2) and influenza A infected pneumonia. MedComm. 2020;1(2):240-8.

23. Tan HB, Xiong F, Jiang YL, Huang WC, Wang Y, Li HH, et al. The study of automatic machine learning base on radiomics of non-focus area in the first chest CT of different clinical types of COVID-19 pneumonia. Sci Rep. 2020;10(1):18926.

24. Jin C, Chen W, Cao Y, Xu Z, Tan Z, Zhang X, et al. Development and evaluation of an artificial intelligence system for COVID-19 diagnosis. Nat Commun. 2020;11(1):5088.

25. Shin KE, Chung MJ, Jung MP, Choe BK, Lee KS. Quantitative computed tomographic indexes in diffuse interstitial lung disease: Correlation with physiologic tests and computed tomography visual scores. Journal of Computer Assisted Tomography. 2011;35(2):266-71.

26. Kauczor HU, Heitmann K, Heussel CP, Marwede D, Uthmann T, Thelen M. Automatic detection and quantification of ground-glass opacities on high-resolution CT using multiple neural networks: comparison with a density mask. AJR Am J Roentgenol. 2000;175(5):1329-34. 
27. LaLonde R, Bagci U. Capsules for object segmentation. arXiv 2018. arXiv preprint arXiv: 180404241.

28. Cox RW. AFNl: software for analysis and visualization of functional magnetic resonance neuroimages. Comput Biomed Res. 1996;29(3):162-73.

29. van Griethuysen JJM, Fedorov A, Parmar C, Hosny A, Aucoin N, Narayan V, et al. Computational Radiomics System to Decode the Radiographic Phenotype. Cancer Res. 2017;77(21):e104-e7.

30. Magidson J. Correlated Component Regression: Re-thinking Regression in the Presence of Near Collinearity. New Perspectives in Partial Least Squares and Related Methods. Springer Proceedings in Mathematics \& Statistics2013. p. 65-78.

31. Traverso A, Wee L, Dekker A, Gillies R. Repeatability and Reproducibility of Radiomic Features: A Systematic Review. Int J Radiat Oncol Biol Phys. 2018;102(4):1143-58.

32. Wold S, Ruhe A, Wold H, Dunn IWJ. The Collinearity Problem in Linear Regression. The Partial Least Squares (PLS) Approach to Generalized Inverses. SIAM Journal on Scientific and Statistical Computing. 1984;5(3):735-43.

33. Abdi H, Williams LJ. Partial Least Squares Methods: Partial Least Squares Correlation and Partial Least Square Regression. Computational Toxicology. Methods in Molecular Biology2013. p. 549-79.

34. Chiarelli AM, Romani GL, Merla A. Fast optical signals in the sensorimotor cortex: General Linear Convolution Model applied to multiple source-detector distance-based data. Neuroimage. 2014;85 Pt 1:245-54.

35. Jolliffe IT, Cadima J. Principal component analysis: a review and recent developments. Philos Trans A Math Phys Eng Sci. 2016;374(2065):20150202.

36. Barlow HB. Unsupervised Learning. Neural Computation. 1989;1(3):295-311.

37. Liu R, Gillies DF. Overfitting in linear feature extraction for classification of high-dimensional image data. Pattern Recognition. 2016;53:73-86.

38. Kearns M, Ron D. Algorithmic stability and sanity-check bounds for leave-one-out cross-validation. Neural Comput. 1999;11(6):1427-53.

39. Chiarelli AM, Croce P, Assenza G, Merla A, Granata G, Giannantoni NM, et al. ElectroencephalographyDerived Prognosis of Functional Recovery in Acute Stroke Through Machine Learning Approaches. International Journal of Neural Systems. 2020.

40. Herpe G, Lederlin M, Naudin M, Ohana M, Chaumoitre K, Gregory J, et al. Efficacy of Chest CT for COVID-19 Pneumonia in France. Radiology. 2020:202568.

41. World Health 0 . Use of chest imaging in COVID-19: a rapid advice guide: web annex A: imaging for COVID-19: a rapid review. Geneva: World Health Organization; 2020 2020. Contract No.: WHO/2019nCoV/Clinical/Radiology_imaging/Web_Annex_A/2020.1.

42. Bradley B, Branley HM, Egan JJ, Greaves MS, Hansell DM, Harrison NK, et al. Interstitial lung disease guideline: the British Thoracic Society in collaboration with the Thoracic Society of Australia and New Zealand and the Irish Thoracic Society. Thorax. 2008;63 Suppl 5:v1-58.

43. The Lancet Infectious D. Curing COVID-19. Lancet Infect Dis. 2020;20(10):1101. 
44. Li W, Wang X, Zhang Y, Li X, Li Q, Ye Z. Radiomic analysis of pulmonary ground-glass opacity nodules for distinction of preinvasive lesions, invasive pulmonary adenocarcinoma and minimally invasive adenocarcinoma based on quantitative texture analysis of CT. Chinese Journal of Cancer Research. 2018.

45. Chae HD, Park CM, Park SJ, Lee SM, Kim KG, Goo JM. Computerized texture analysis of persistent part-solid ground-glass nodules: Differentiation of preinvasive lesions from invasive pulmonary adenocarcinomas. 2014.

\section{Figures}


Patients with proved SARS-CoV-2 (COVID-19) underwent Chest CT between March 2020 and April 2020

$$
(n=120)
$$

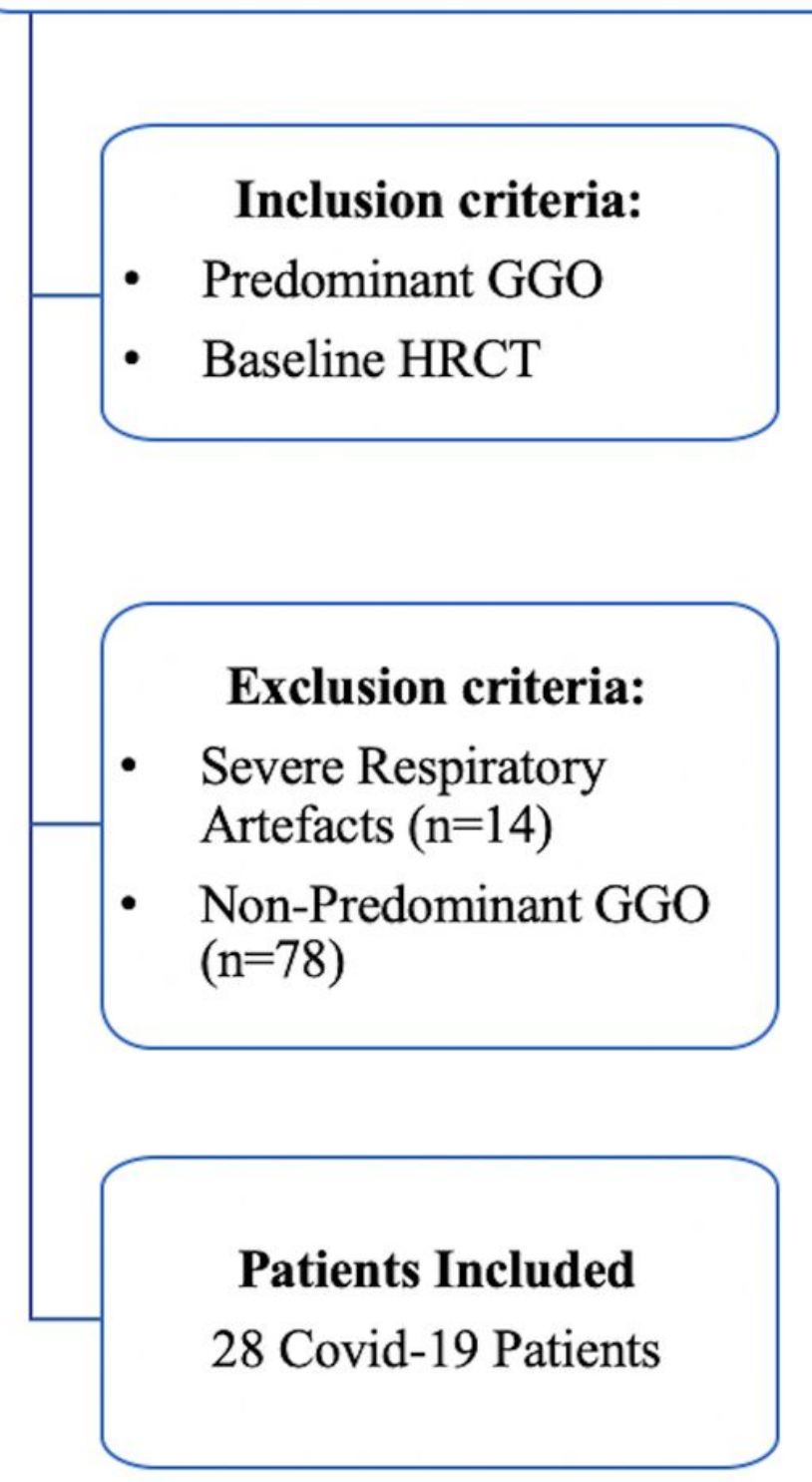

Patients with proved acute respiratory disease (nCOVID-19) underwent Chest CT between August 2019 and April 2020

$$
(n=310)
$$

\section{Inclusion criteria:}

- Predominant GGO

- Availability of Final Diagnosis

\section{Exclusion criteria:}

- Severe Respiratory Artefacts $(\mathrm{n}=32)$

- Non-Predominant GGO $(\mathrm{n}=210)$

- Final diagnosis not available $(\mathrm{n}=38)$

\section{Patients Included} 30 nCovid-19 Patients

Figure 1

Study Flowchart. 
Patients with proved SARS-CoV-2 (COVID-19) underwent Chest CT between March 2020 and April 2020

$$
(n=120)
$$

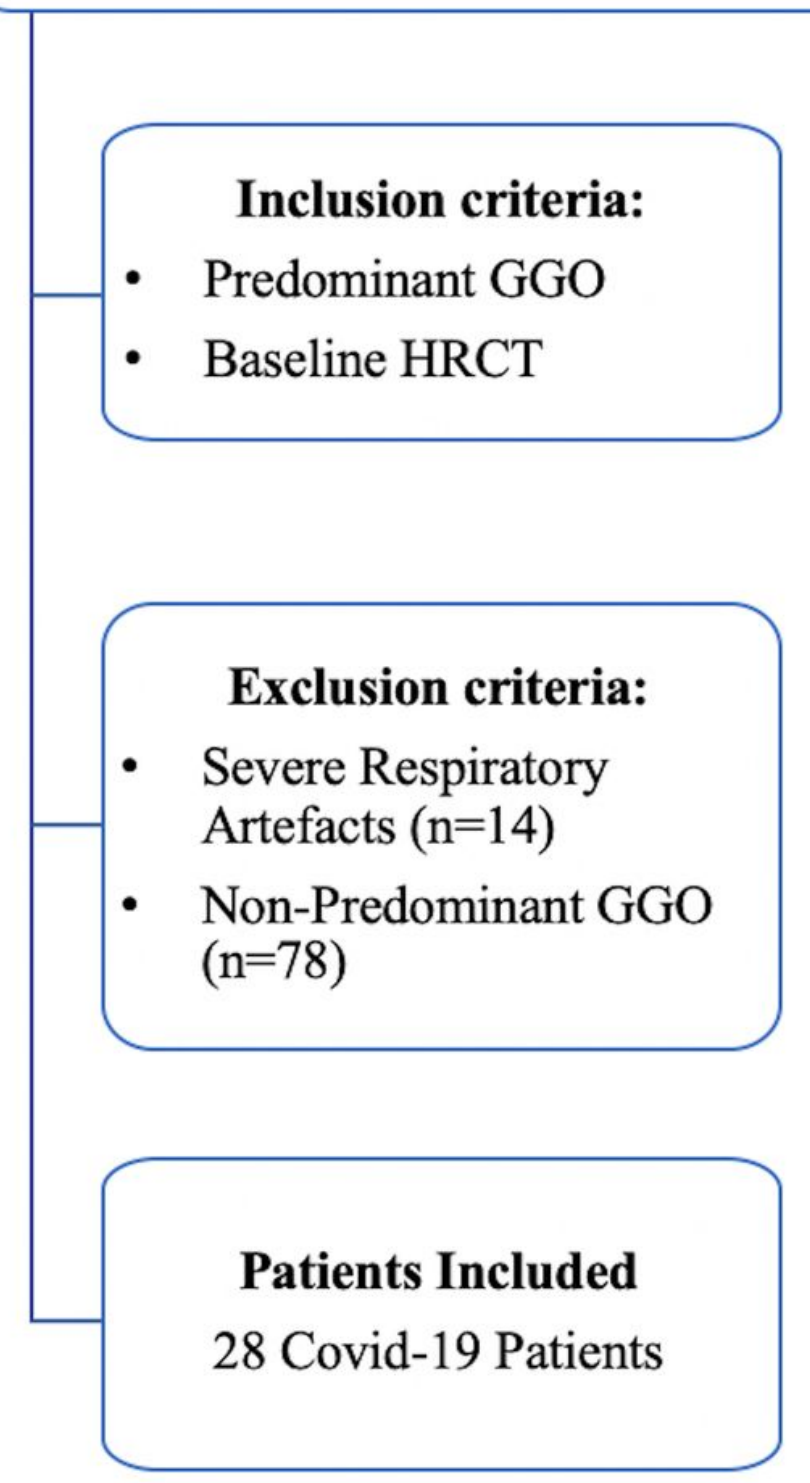

Patients with proved acute respiratory disease (nCOVID-19) underwent Chest CT between August 2019 and April 2020

$$
(\mathrm{n}=310)
$$

\section{Inclusion criteria:}

- Predominant GGO

- Availability of Final Diagnosis

\section{Exclusion criteria:}

- Severe Respiratory Artefacts $(\mathrm{n}=32)$

- Non-Predominant GGO $(\mathrm{n}=210)$

- Final diagnosis not available $(\mathrm{n}=38)$

\section{Patients Included} 30 nCovid-19 Patients

Figure 1

Study Flowchart. 


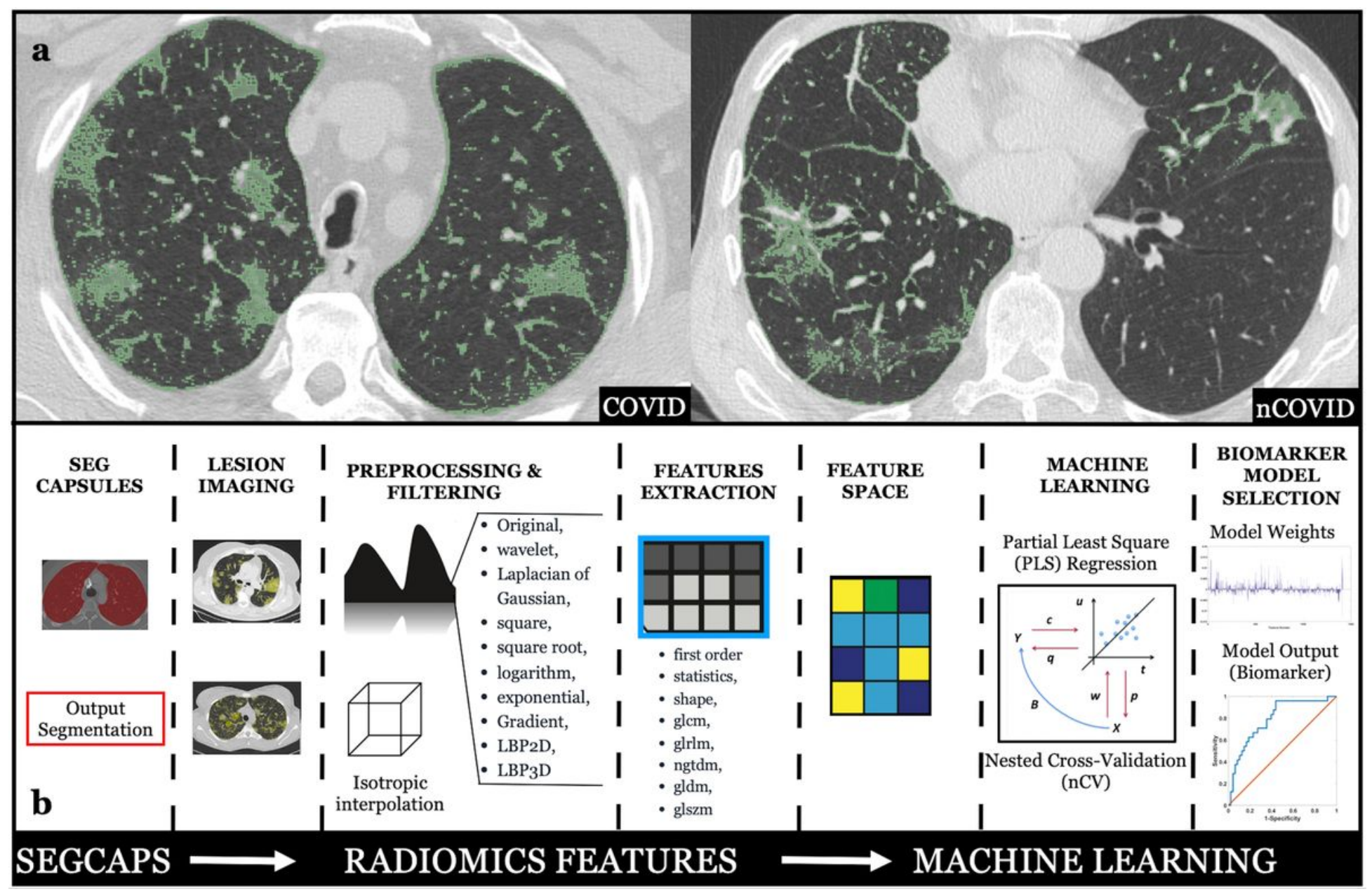

Figure 2

(a) GGO segmentations of COVID-19 pneumonia (COVID) and non-COVID-19 acute lung disease (nCOVID) on chest HRCT. (b) Schematic representation of the radiomic features extraction and the machine learning framework implemented. 


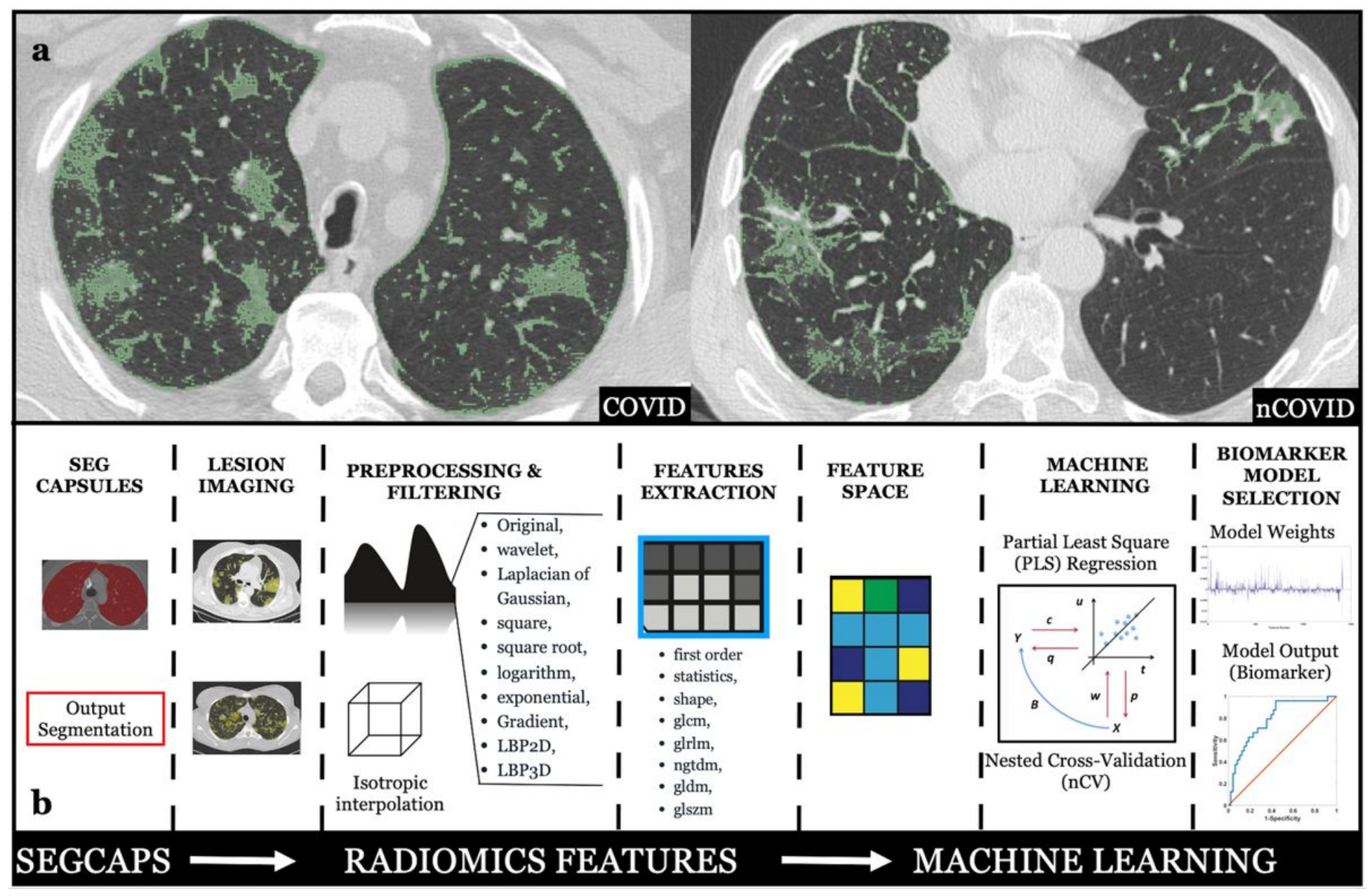

Figure 2

(a) GGO segmentations of COVID-19 pneumonia (COVID) and non-COVID-19 acute lung disease (nCOVID) on chest HRCT. (b) Schematic representation of the radiomic features extraction and the machine learning framework implemented. 


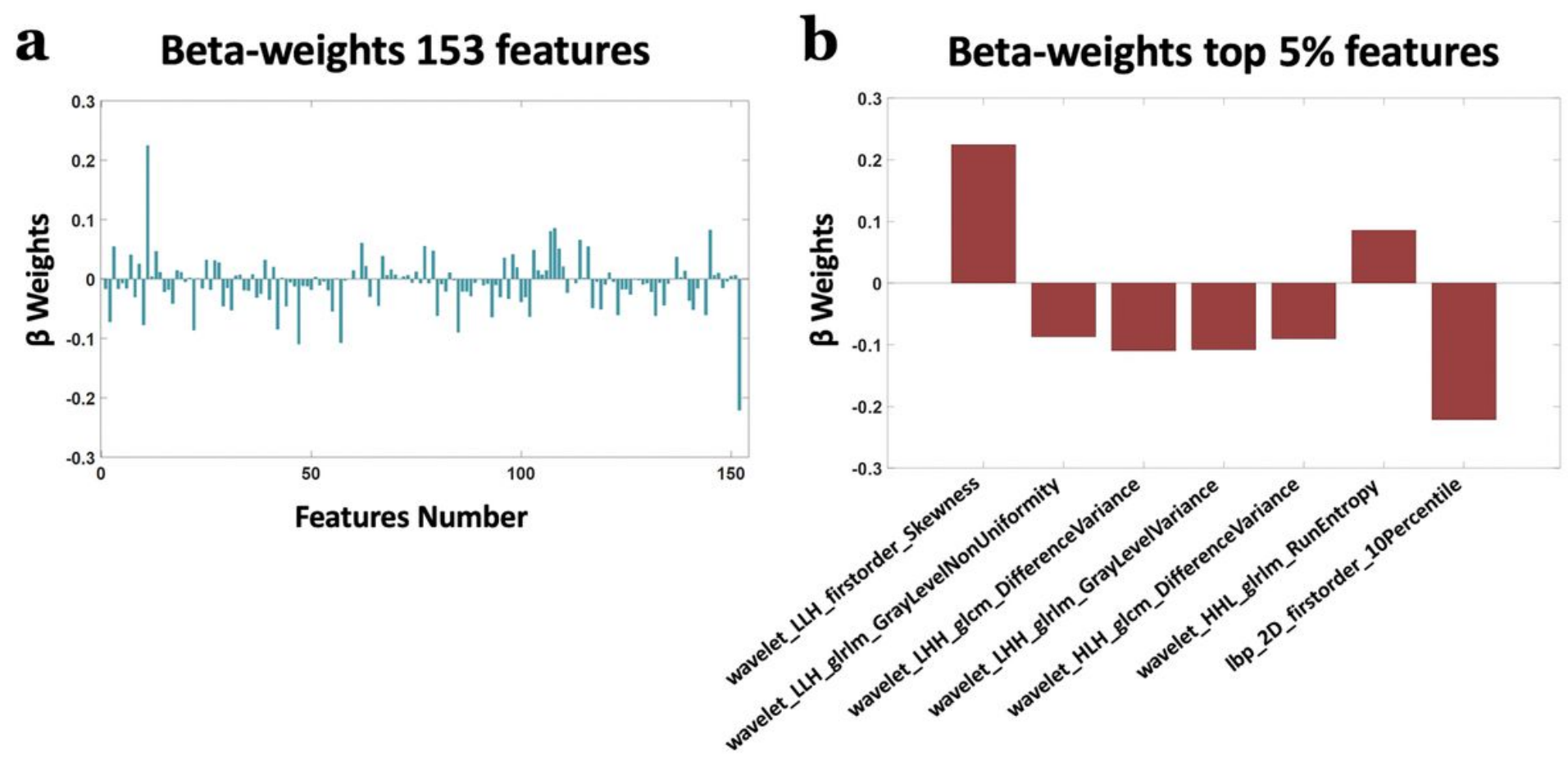

Figure 3

ROC analysis of the machine learning (PLS) classification performance (COVID patients were labelled as "positive", nCOVID patients as "negative").

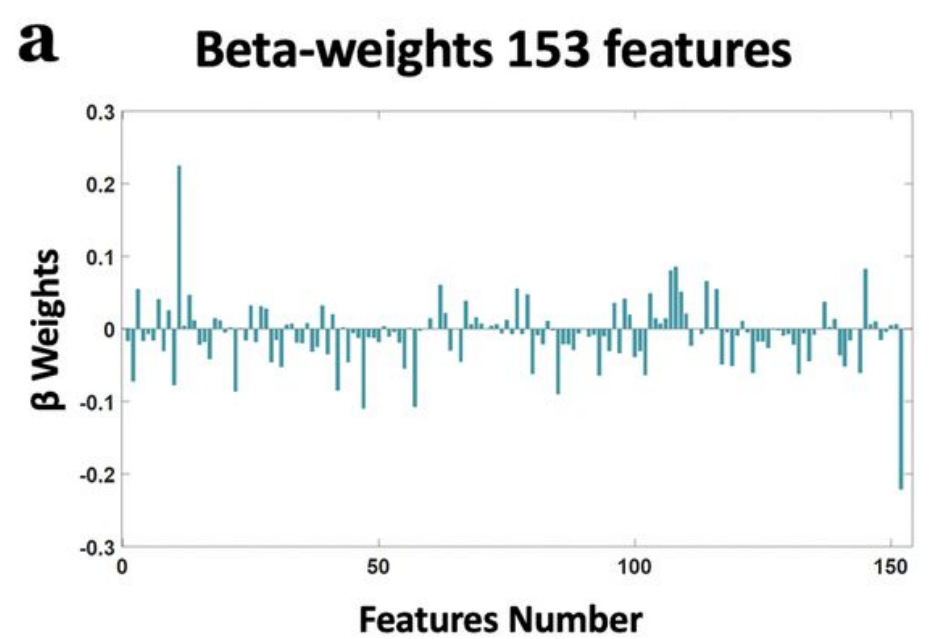

b Beta-weights top $5 \%$ features

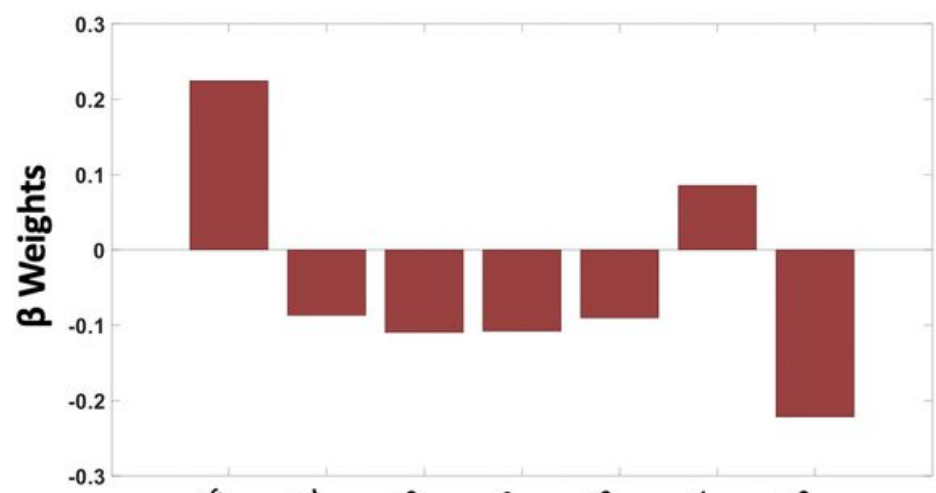

Features Number

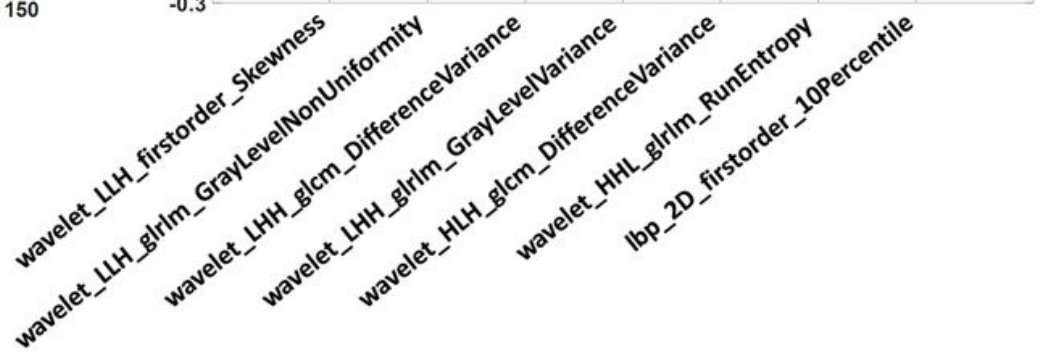

Figure 3

ROC analysis of the machine learning (PLS) classification performance (COVID patients were labelled as "positive", nCOVID patients as "negative"). 


\section{Radiomic Features}

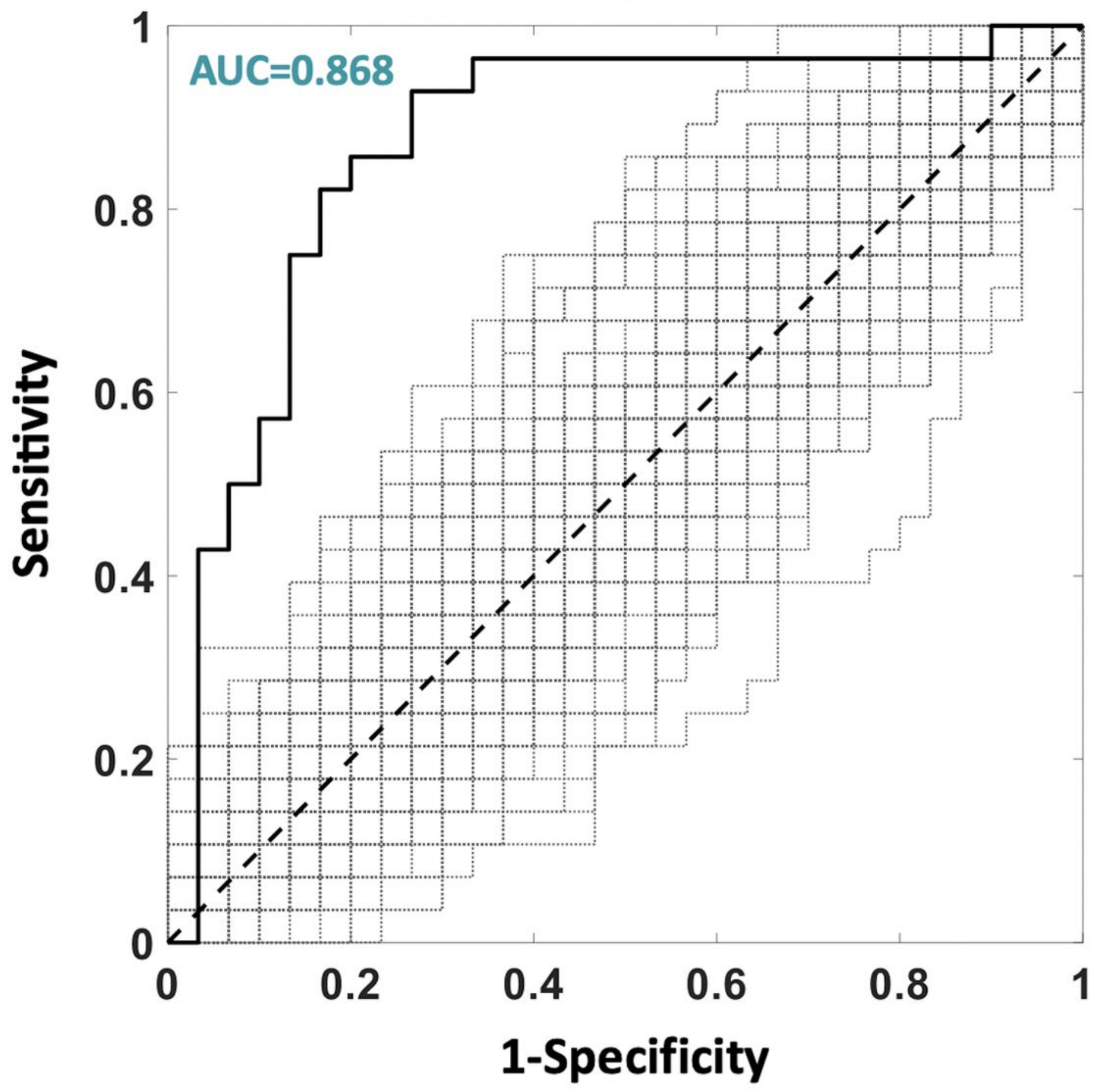

Figure 4

(a) PLS $\beta$-weights associated with reliable (r>0.95) radiomic features. (b) $\beta$-weights associated to the top $5 \%$ of features with the largest $\beta$-weights in magnitude, i.e. those most impacting the differential diagnosis. 


\section{Radiomic Features}



Figure 4

(a) PLS $\beta$-weights associated with reliable (r>0.95) radiomic features. (b) $\beta$-weights associated to the top $5 \%$ of features with the largest $\beta$-weights in magnitude, i.e. those most impacting the differential diagnosis. 